Departamento de Enfermedades Respiratorias, Facultad de Medicina, Pontificia Universidad Católica de Chile. Santiago, Chile. aAlumnos de Medicina, Facultad de Medicina, Pontificia Universidad Católica de Chile. Santiago, Chile.

Fuente de financiamiento: Proyectos Fondecyt 1080671 y 1141108.

Autores declaran no tener conflictos de interés.

Recibido el 7 de noviembre de 2017, aceptado el 3 de abril de 2018.

Correspondencia a: Dr. Fernando Saldías Peñafiel Departamento de Enfermedades Respiratorias. División de Medicina, Facultad de Medicina. Pontificia Universidad Católica de Chile.

Teléfonos: (562) 26331541 (562) 23543242 Fax: (562) 26335255 Diagonal Paraguay 362 Sexto Piso, Santiago, Chile. fsaldias@med.puc.cl

\section{Variables clínicas y funcionales asociadas al riesgo de muerte en el seguimiento a largo plazo en pacientes con enfermedad pulmonar obstructiva crónica}

\author{
FERNANDO SALDÍAS, JAVIERA GASSMANN \\ ALEJANDRO CANELOa, ORLANDO DÍAZ
}

Background: Identifying risk factors for long-term mortality in patients with chronic obstructive pulmonary disease (COPD) could improve their clinical management. Aim: To examine the clinical variables associated to long-term mortality in a cohort of COPD patients. Patients and Methods: A clinical and respiratory functional assessment, chest computed tomography and clinical follow up for five years was carried out in 202 COPD patients aged $66 \pm 9$ years (59\% males), active or former smokers of 10 or more pack-years. Results: Thirty four percent of patients were active smokers, consuming $46 \pm 23$ packs/year, $86 \%$ had comorbidities, especially chronic cardiovascular and metabolic diseases. Forty-six patients died in the five years follow-up (5-year mortality was therefore 22.8\%). In the univariate analysis, the main risk factors associated to long-term mortality were an older age, male sex, dyspnea severity, severe exacerbation risk, chronic respiratory failure, magnitude of lung emphysema, airflow obstruction and lung hyperinflation, reduction of thigh muscle cross-sectional area and physical activity limitation. In the multivariate analysis, the three independent risk factors for long-term mortality were dyspnea severity, chronic hypoxemia and exercise limitation measured with the six minutes' walk test. Conclusions: Systematic clinical assessment allowed to identify the main risk factors associated with long-term mortality in patients with COPD, which could be used in planning preventive and management programs aimed at the high-risk population.

(Rev Med Chile 2018; 146: 422-432)

Key words: Pulmonary Disease, Chronic Obstructive; Mortality; Prognosis; Risk Factors.

L a enfermedad pulmonar obstructiva crónica (EPOC), según la definición de la Iniciativa Global para la Enfermedad Pulmonar Obstructiva Crónica (GOLD), es una enfermedad común, prevenible y tratable, caracterizada por síntomas respiratorios persistentes y limitación crónica del flujo aéreo que generalmente es pro- gresiva y está asociada a una respuesta inflamatoria anormal que afecta a la vía aérea y parénquima pulmonar ${ }^{1}$. Esta enfermedad es una de las principales causas de morbilidad y mortalidad en el mundo, actualmente es la tercera causa de muerte y representa una enorme carga económica para la sociedad ${ }^{1-3}$. Se estima que afecta a $6 \%$ de la 
población adulta en Estados Unidos de Norteamérica y a $14,5 \%$ de los adultos mayores de 40 años en Chile ${ }^{4,5}$. La exacerbación de la EPOC se caracteriza por un empeoramiento agudo de los síntomas respiratorios más allá de las fluctuaciones diarias que conduce a modificación de la terapia farmacológica ${ }^{6}$. La historia natural de la EPOC está marcada por las exacerbaciones que representan la mayor parte de la carga económica, deterioro de la calidad de vida de los enfermos y demanda de atención en los servicios de salud $\mathrm{d}^{6}$.

La obstrucción progresiva del flujo aéreo que es característica de la EPOC está estrechamente relacionada con su morbilidad y mortalidad ${ }^{7-9}$. Por lo tanto, el grado de obstrucción al flujo aéreo generalmente se considera el factor clave para evaluar la gravedad de la EPOC y para guiar y monitorear el tratamiento ${ }^{1}$. Sin embargo, las intervenciones farmacológicas (agonistas $\beta 2$-adrenérgicos, anticolinérgicos y corticoides) no han logrado modificar la progresión de la obstrucción del flujo aéreo ni reducir la mortalidad prematura asociada a esta condición ${ }^{10-12}$. En la actualidad, se recomienda considerar la magnitud de la disnea o limitación de la actividad física, grado de obstrucción bronquial, las comorbilidades y el riesgo de exacerbaciones en el manejo de la enfermedad, reconociendo su condición multidimensional que comprende varios fenotipos ${ }^{13-16}$. Además, los pacientes suelen fallecer por otras condiciones distintas a la enfermedad de base, tales como enfermedad cardiovascular o cáncer, especialmente en las etapas precoces de la enfermedad ${ }^{17}$. Por lo tanto, la estadificación de la gravedad y el tratamiento de la EPOC deben tomar en consideración la naturaleza heterogénea y multidimensional de la enfermedad ${ }^{1}$. El propósito de este estudio es examinar las variables clínicas y funcionales asociadas al riesgo de muerte en el largo plazo en una cohorte de pacientes con enfermedad pulmonar obstructiva crónica.

\section{Pacientes y Métodos}

Estudio clínico descriptivo prospectivo de seguimiento a cinco años (enero de 2010 a diciembre de 2015) que examinó las variables clínicas, imagenológicas y funcionales asociadas al riesgo de muerte en el seguimiento a largo plazo en pacientes con EPOC tabáquica atendidos en la red de salud de la Universidad Católica. Se evaluaron
202 pacientes fumadores o exfumadores portadores de EPOC tabáquica, definida de acuerdo a los criterios de $\mathrm{GOLD}^{1}$, en condiciones estables de su enfermedad, quienes aceptaron participar en el estudio clínico firmando un acta de consentimiento informado aprobada por el Comité de Ética en Investigación de la institución. Los criterios de inclusión fueron: adultos mayores de 40 años con EPOC ( $\mathrm{VEF}_{1} / \mathrm{CVF}$ posterior a broncodilatador menor a 70\%), con historia de tabaquismo de 10 o más paquetes año, ser fumador activo o haber dejado de fumar en los últimos veinte años y no tener alguna enfermedad que les impidiera realizar la evaluación funcional respiratoria en el momento del ingreso al estudio. Se excluyó a los pacientes con historia de asma bronquial, bronquiectasias, fibrosis pulmonar, secuelas de tuberculosis, enfermedad neoplásica activa, vasculitis, inmunodeficiencia, comorbilidades descompensadas o resección pulmonar.

\section{Evaluación clínica}

En los pacientes incorporados en el estudio se consignó la edad, sexo, ocupación, consumo de tabaco (expresado en paquetes/año), condición de fumador activo o exfumador, tiempo de suspensión del tabaquismo, comorbilidades, tratamiento farmacológico y uso de oxígeno domiciliario, peso, talla e índice de masa corporal (IMC), se evaluó la magnitud de la disnea utilizando la escala de disnea modificada del Medical Research Council del Reino Unido ${ }^{18}$ (mMRC), historia de tos y expectoración crónica, y el número de exacerbaciones en los últimos 12 meses, de acuerdo a la definición de Burge y Wedzicha ${ }^{19}$. La información de sobrevida en el seguimiento a cinco años fue obtenida de los registros clínicos y el Servicio de Registro Civil e Identificación.

\section{Evaluación funcional respiratoria}

Se realizó una espirometría con un equipo Sensor Medics 2200 (CareFusion, Yorba Linda, CA, EE. UU.) antes y después de la administración de $400 \mu \mathrm{g}$ de salbutamol, siguiendo las normas de las sociedades Americana de Tórax (ATS) y Europea de Enfermedades Respiratorias (ERS) ${ }^{20}$, empleando los valores de referencia de Hankinson y cols ${ }^{21}$. Se midió los volúmenes pulmonares (capacidad pulmonar total [CPT], capacidad residual funcional $[\mathrm{CRF}]$, volumen residual $[\mathrm{VR}]$ y capacidad inspiratoria $[\mathrm{CI}]$ ) en un pletismógrafo 
corporal Vmax Autobox V62J de BD Medical Supplies (Vyaire Medical, Mettawa, IL, EE.UU.), siguiendo las recomendaciones de la ATS/ERS ${ }^{22}$. Se midió la capacidad de difusión de monóxido de carbono $\left(\mathrm{DL}_{\mathrm{CO}}\right)$ siguiendo las recomendaciones de la ATS/ERS ${ }^{23}$, empleando los valores de referencia de Cotes y cols ${ }^{24}$. Se consignó la presencia de insuficiencia respiratoria parcial o global mediante gasometría arterial. Se efectuó una prueba de caminata en 6 min de acuerdo a las normas de la $\mathrm{ATS}^{25}$. Durante la caminata se midió la saturación arterial de oxígeno $\left(\mathrm{SpO}_{2}\right)$ y frecuencia cardíaca con oxímetro de pulso (Nonin Medical Inc., Plymouth, Mn., EE. UU.). La disnea y fatigabilidad de las piernas se evaluaron empleando la escala psicofísica de Borg ${ }^{26}$. En esta prueba se emplearon los valores de referencia de Troosters y $\mathrm{cols}^{27}$. Se calculó el índice de gravedad multidimensional $\mathrm{BODE}^{28}$, que incluye el IMC, $\mathrm{VEF}_{1}$, magnitud de la disnea y distancia recorrida en $6 \mathrm{~min}$.

\section{Estudio de imágenes}

Se realizó una tomografía helicoidal de tórax convencional en todos los pacientes al ser enrolados en el estudio. Un médico radiólogo involucrado en el estudio examinó las imágenes, consignando la magnitud del enfisema pulmonar, definido como el porcentaje de áreas de baja atenuación inferior a 960 unidades Hounsfield ${ }^{29}$ y el área de sección transversal del músculo del muslo derecho siguiendo las recomendaciones de Bernard y $\operatorname{cols}^{30}$. El examen tomográfico se realizó con el paciente en inspiración profunda, con un equipo multidetector de 64 canales (Somatom Sensation 64, Siemens Healthcare, Erlangen, Alemania), que fue calibrado diariamente para la densidad tomográfica del aire y cada tres meses para el agua. El protocolo de adquisición de imágenes fue: $120 \mathrm{kVp}, 200 \mathrm{mAs}$ y tiempo de rotación de 0,33 segundos. Las imágenes fueron reconstruidas usando un algoritmo de $1 \mathrm{~mm}$ de grosor de corte y $0,7 \mathrm{~mm}$ de intervalo.

\section{Análisis estadístico}

Los resultados son expresados como valores promedio \pm desviación estándar para las variables medidas en escala numérica y en porcentaje para las medidas en escala nominal. Las variables cualitativas fueron comparadas mediante la prueba de $\chi^{2}$ o test exacto de Fisher y las variables continuas con la prueba t de Student. Las variables clínicas y funcionales asociadas al riesgo de muerte en el seguimiento a cinco años fueron sometidas a análisis univariado y multivariado en un modelo de regresión logística (modalidad stepwise) que permite el control simultáneo de múltiples factores. De este modo, los parámetros que no agregaron valor predictivo no fueron retenidos en el modelo. $\mathrm{El}$ análisis de las curvas receptor operador de las variables clínicas y funcionales permitió estimar los puntos de corte y su capacidad predictiva de la mortalidad (AUC: Área bajo la curva). Se calcularon las razones de probabilidad (odds ratio) e intervalos de confianza (IC) para 95\%. Las diferencias entre las variables fueron consideradas significativas con un valor de $\mathrm{p}<0,05$.

\section{Resultados}

Se enrolaron 202 pacientes con enfermedad pulmonar obstructiva crónica tabáquica, edad promedio: $66 \pm 9$ años (rango: $42-88$ ), 59\% eran varones, un tercio eran fumadores activos, consumo de tabaco promedio: $46 \pm 23$ paq/año, $57 \%$ referían tos y expectoración crónica y disnea $\geq 2$ de la escala mMRC, 39\% correspondía a las categorías 3 y 4 de GOLD, 86\% tenía comorbilidades, especialmente cardiovasculares y metabólicas crónicas (Tabla 1). Dos tercios de los pacientes estaban siendo tratados con $\beta_{2}$-adrenérgicos, anticolinérgicos de acción prolongada o ambos, $58 \%$ con corticoides inhalados y todos estaban siendo manejados con broncodilatadores de acción corta según necesidad. El 58\% de los pacientes había presentado exacerbaciones de la EPOC en los últimos doce meses, siendo $20 \%$ de ellas exacerbaciones graves manejadas en el hospital. Cuarenta y seis pacientes fallecieron en el seguimiento a cinco años $(22,8 \%)$.

Los pacientes con EPOC que fallecieron en el seguimiento a cinco años eran de edad más avanzada, predominio sexo masculino, presentaban mayor deterioro clínico (referían mayor magnitud de la disnea según la escala mMRC y exacerbaciones graves en los últimos doce meses) $\mathrm{y}$ funcional (insuficiencia respiratoria parcial o global, mayor obstrucción bronquial y signos de hiperinsuflación pulmonar, reducción de la $\mathrm{DL}_{\mathrm{CO}}$, área de sección transversal del músculo del muslo y mayor limitación en la actividad física) (Tabla 2).

En el análisis univariado, las principales variables clínicas asociadas al riesgo de muerte en 
Variables asociadas a riesgo de muerte en seguimiento de EPOC - F. Saldías et al

Tabla 1. Características clínicas y funcionales de la cohorte de pacientes con enfermedad pulmonar obstructiva crónica

\begin{tabular}{|c|c|c|}
\hline Características & Número $(\bar{x} \pm D E)$ & Porcentaje (Rango) \\
\hline $\mathrm{n}$ & 202 & $100 \%$ \\
\hline Edad (años) & $66,4 \pm 8,8$ & (R: $42-88)$ \\
\hline Sexo (Masculino-Femenino) & $120-82$ & $59,4-40,6$ \\
\hline Tabaquismo (Exfumador - Fumador activo) & $133-69$ & $65,8-34,2$ \\
\hline Consumo de tabaco (paq/año) & $46,4 \pm 23,0$ & $(R: 10-170)$ \\
\hline Tos y expectoración crónica & 115 & 56,9 \\
\hline Disnea $(m M R C \geq 2)$ & 117 & 57,9 \\
\hline Área de enfisema (\% -960 UH) & $15,9 \pm 10,7$ & $(R: 0,6-60,6)$ \\
\hline Área de sección del músculo del muslo $\left(\mathrm{cm}^{2}\right)$ & $59,8 \pm 18,4$ & (R: $8,0-110,6)$ \\
\hline Exacerbaciones de la EPOC $\leq 12$ meses & $1,4 \pm 1,8$ & $(R: 0-10)$ \\
\hline Índice BODE & $2,2 \pm 2,1$ & $(\mathrm{R}: 0-8)$ \\
\hline Hipoxemia $\left(\mathrm{PaO}_{2}<60 \mathrm{mmHg}\right)$ & $27 / 183$ & 14,8 \\
\hline Hipercapnia $\left(\mathrm{PaCO}_{2}>45 \mathrm{mmHg}\right)$ & $28 / 183$ & 15,3 \\
\hline Índice de masa corporal-IMC $\left(\mathrm{kg} / \mathrm{m}^{2}\right)$ & $26,4 \pm 4,3$ & (R: $14,6-38,4)$ \\
\hline Comorbilidades & $174 / 202$ & 86,11 \\
\hline Cardiopatía coronaria & 18 & 8,9 \\
\hline Hipertensión arterial & 110 & 54,5 \\
\hline Diabetes mellitus & 31 & 15,3 \\
\hline Dislipidemia & 75 & 37,1 \\
\hline Reflujo gastroesofágico & 67 & 33,2 \\
\hline Obesidad (IMC > $\left.30 \mathrm{~kg} / \mathrm{m}^{2}\right)$ & 40 & 19,8 \\
\hline Depresión & 29 & 14,4 \\
\hline Capacidad vital forzada-CVF (mL) & $3.268 \pm 1.047$ & (98 \pm 21\% teórico) \\
\hline Volumen espiratorio forzado $1^{\text {er }}$ seg- $-V_{E F}(\mathrm{~mL})$ & $1.601 \pm 782$ & (62 $\pm 25 \%$ teórico) \\
\hline $\mathrm{VEF}_{1} / \mathrm{CVF}(\%)$ & $48,3 \pm 14,9$ & (R: $16-69)$ \\
\hline $\mathrm{DL}_{\mathrm{CO}}(\mathrm{mL} / \mathrm{min} / \mathrm{mmHg})$ & $16,0 \pm 6,3$ & (71 \pm 23\% teórico) \\
\hline $\mathrm{DL}_{\mathrm{co}} \mathrm{NA}(\mathrm{mL} / \mathrm{min} / \mathrm{mmHg} / \mathrm{L})$ & $3,5 \pm 0,9$ & (87 $\pm 23 \%$ teórico) \\
\hline Capacidad pulmonar total-CPT (mL) & $6.489 \pm 1.489$ & (120 $\pm 21 \%$ teórico) \\
\hline Capacidad inspiratoria-Cl (mL) & $2.306 \pm 813$ & (88 \pm 24\% teórico) \\
\hline Capacidad residual funcional-CRF ( $\mathrm{mL})$ & $4.158 \pm 1.284$ & (136 \pm 39\% teórico) \\
\hline Volumen residual-VR (mL) & $3.102 \pm 1.104$ & $(145 \pm 54 \%$ teórico $)$ \\
\hline $\mathrm{Cl} / \mathrm{CPT}$ & $36,2 \pm 10,6$ & $(R: 6,9-64,1)$ \\
\hline CRF/CPT & $63,5 \pm 10,5$ & $(R: 35,9-92,5)$ \\
\hline VR/CPT & $47,5 \pm 11,3$ & (R: $22,0-82,4)$ \\
\hline Distancia recorrida en 6 min (m) & $467 \pm 103$ & (79 $\pm 16 \%$ teórico) \\
\hline Sobrevida media a 60 meses & $54,1 \pm 13,9$ & $(R: 2-60)$ \\
\hline Fallecidos en seguimiento a 60 meses & $46 / 202$ & 22,8 \\
\hline
\end{tabular}

Nota: $\overline{\mathrm{x}} \pm$ DE: Promedio \pm Desviación estándar. EPOC: Enfermedad pulmonar obstructiva crónica; $\mathrm{DL}_{\mathrm{cO}}$ : Capacidad de difusión de monóxido de carbono; VA: Volumen alveolar. 


\section{Tabla 2. Características clínicas y funcionales de la cohorte de pacientes con enfermedad pulmonar} obstructiva crónica según sobrevida en el seguimiento a cinco años

\begin{tabular}{|c|c|c|c|}
\hline Características & Vivos & Fallecidos & $\mathbf{p}$ \\
\hline $\mathrm{n}$ & 156 & 46 & \\
\hline Edad (años) & $65,5 \pm 8,6$ & $69,4 \pm 8,9$ & 0,008 \\
\hline Sexo (Masculino-Femenino) & $87-69$ & $33-13$ & 0,053 \\
\hline Fumador activo & $57(36,5 \%)$ & $12(26,1 \%)$ & 0,190 \\
\hline Consumo de tabaco (paq/año) & $46,6 \pm 23,7$ & $45,6 \pm 20,9$ & 0,796 \\
\hline Tos y expectoración crónica & $86(55,1 \%)$ & $29(63,0 \%)$ & 0,342 \\
\hline Disnea $(m M R C \geq 2)$ & $80(51,3 \%)$ & $37(80,4 \%)$ & 0,001 \\
\hline Categorías de GOLD & $2,1 \pm 0,9$ & $2,7 \pm 1,1$ & 0,001 \\
\hline Índice BODE & $1,9 \pm 1,9$ & $3,4 \pm 2,3$ & 0,001 \\
\hline Exacerbaciones de la EPOC $\leq 12$ meses & $88(56,4 \%)$ & $29(63,0 \%)$ & 0,424 \\
\hline Exacerbaciones graves de la EPOC $\leq 12$ meses & $15(9,6 \%)$ & $10(21,7 \%)$ & 0,029 \\
\hline Tratamiento con oxígeno domiciliario & $5(3,2 \%)$ & $10(21,7 \%)$ & 0,001 \\
\hline Hipoxemia $\left(\mathrm{PaO}_{2}<60 \mathrm{mmHg}\right)$ & $11(7,7 \%)$ & $16(40 \%)$ & 0,001 \\
\hline Hipercapnia $\left(\mathrm{PaCO}_{2}>45 \mathrm{mmHg}\right)$ & $14(9,8 \%)$ & $14(35 \%)$ & 0,001 \\
\hline Índice de masa corporal (kg/m²) & $26,4 \pm 4,1$ & $26,2 \pm 4,8$ & 0,782 \\
\hline Comorbilidades & $137(87,8 \%)$ & $37(80,4 \%)$ & 0,204 \\
\hline Capacidad vital forzada-CVF (mL) & $3.384 \pm 1.045$ & $2.876 \pm 962$ & 0,004 \\
\hline Capacidad vital forzada (\% teórico) & $100,6 \pm 20,0$ & $89,0 \pm 24,2$ & 0,001 \\
\hline Volumen espiratorio forzado $1^{\mathrm{er}}$ seg-VEF $(\mathrm{mL})$ & $1.678 \pm 771$ & $1.342 \pm 771$ & 0,010 \\
\hline $\operatorname{VEF}_{1}$ (\% teórico) & $64,2 \pm 23,9$ & $53,6 \pm 27,8$ & 0,012 \\
\hline Relación VEF $/$ /CVF (\%) & $49,3 \pm 14,6$ & $45,0 \pm 15,6$ & 0,086 \\
\hline $\mathrm{DL}_{\mathrm{co}}(\mathrm{mL} / \mathrm{min} / \mathrm{mmHg})$ & $16,6 \pm 6,3$ & $12,9 \pm 5,2$ & 0,004 \\
\hline $\mathrm{DL}_{\mathrm{co}}(\%$ teórico $)$ & $73,8 \pm 22,6$ & $58,8 \pm 23,8$ & 0,002 \\
\hline $\mathrm{DL}_{\mathrm{co}} \mathrm{NA}(\mathrm{mL} / \mathrm{min} / \mathrm{mmHg} / \mathrm{L})$ & $3,6 \pm 0,9$ & $3,1 \pm 0,9$ & 0,007 \\
\hline Capacidad pulmonar total-CPT (mL) & $6.445 \pm 1.535$ & $6.659 \pm 1.310$ & 0,458 \\
\hline CPT (\% teórico) & $119,0 \pm 19,5$ & $124,3 \pm 24,7$ & 0,186 \\
\hline Capacidad inspiratoria- $\mathrm{Cl}(\mathrm{mL})$ & $2.405 \pm 819$ & $1.943 \pm 682$ & 0,003 \\
\hline Cl (\% teórico) & $90,6 \pm 22,9$ & $77,9 \pm 26,0$ & 0,006 \\
\hline Capacidad residual funcional-CRF $(\mathrm{mL})$ & $4.025 \pm 1.232$ & $4.663 \pm 1.370$ & 0,009 \\
\hline CRF (\% teórico) & $131,7 \pm 34,9$ & $152,7 \pm 50,8$ & 0,006 \\
\hline Volumen residual-VR (mL) & $2.958 \pm 1.012$ & $3.646 \pm 1.274$ & 0,002 \\
\hline VR (\% teórico) & $139,8 \pm 47,6$ & $168,6 \pm 69,9$ & 0,006 \\
\hline $\mathrm{Cl} / \mathrm{CPT}$ & $37,7 \pm 10,2$ & $30,4 \pm 10,4$ & 0,001 \\
\hline CRF/CPT & $62,0 \pm 10,0$ & $69,2 \pm 10,5$ & 0,001 \\
\hline $\mathrm{VR} / \mathrm{CPT}$ & $45,8 \pm 10,6$ & $54,0 \pm 11,6$ & 0,001 \\
\hline Distancia recorrida en $6 \min (\mathrm{m})$ & $488 \pm 89$ & $394 \pm 117$ & 0,001 \\
\hline Distancia recorrida en 6 min (\% teórico) & $82,4 \pm 13,3$ & $68,7 \pm 20,7$ & 0,001 \\
\hline Área de enfisema $(\%-960$ UH) & $15,1 \pm 9,9$ & $19,9 \pm 13,1$ & 0,041 \\
\hline Área de sección del músculo del muslo $\left(\mathrm{cm}^{2}\right)$ & $61,3 \pm 17,5$ & $52,6 \pm 20,9$ & 0,032 \\
\hline Sobrevida media a 60 meses & $60,0 \pm 0$ & $34,1 \pm 18,2$ & 0,001 \\
\hline
\end{tabular}

Nota: EPOC: Enfermedad pulmonar obstructiva crónica; $\mathrm{DL}_{\mathrm{co}}$ : Capacidad de difusión de monóxido de carbono; VA: Volumen alveolar. 
Variables asociadas a riesgo de muerte en seguimiento de EPOC - F. Saldías et al

el seguimiento a largo plazo (60 meses) fueron la edad, sexo masculino, magnitud de la disnea, exacerbaciones graves en los últimos doce meses, uso de oxígeno domiciliario, hipoxemia e hipercapnia crónica, gravedad de la obstrucción bronquial (reducción de $\mathrm{VEF}_{1}$ ), magnitud del enfisema (\% -960 UH), signos de hiperinsuflación pulmonar (reducción de la capacidad inspiratoria $\mathrm{y}$ aumento de la capacidad residual funcional y volumen residual), reducción de la $\mathrm{DL}_{\mathrm{CO}}$, área de sección transversal del músculo del muslo y distancia recorrida en seis minutos (Tabla 3). En el análisis multivariado, los principales predictores clínicos de mortalidad independientes fueron la magnitud de la disnea (OR: 1,86; IC95\% 1,30-2,65; $\mathrm{p}<0,001$ ), $\mathrm{PaO}_{2}$ (OR: 0,94; IC95\% 0,88-0,99; p: 0,045$)$ y la distancia recorrida en seis minutos (OR: 0,99; IC95\% 0,98-0,99; p: 0,014) (Tabla 4).

Los principales predictores clínicos y funcionales asociados al riesgo de muerte en el seguimiento a largo plazo en pacientes con EPOC fueron la magnitud de la disnea (AUC: 0,67), $\mathrm{PaO}_{2}$ (AUC:

Tabla 3. Variables clínicas y funcionales asociadas al riesgo de muerte en el seguimiento a cinco años de una cohorte de pacientes con enfermedad pulmonar obstructiva crónica. Análisis univariado

\begin{tabular}{|c|c|c|c|}
\hline Factor pronóstico & Odds ratio & IC $95 \%$ & $\mathbf{p}$ \\
\hline Edad $\geq 65$ años & 2,62 & $1,27-5,44$ & 0,009 \\
\hline Sexo masculino & 2,01 & $0,99-4,12$ & 0,056 \\
\hline Disnea $(m M R C \geq 2)$ & 3,91 & $1,77-8,63$ & 0,001 \\
\hline Exacerbaciones graves de la EPOC $\leq 12$ meses & 2,61 & $1,08-6,29$ & 0,033 \\
\hline Tratamiento con oxígeno domiciliario & 8,39 & $2,70-26,06$ & 0,001 \\
\hline Hipoxemia $\left(\mathrm{PaO}_{2}<60 \mathrm{mmHg}\right)$ & 8,00 & $3,31-19,33$ & 0,001 \\
\hline Hipercapnia $\left(\mathrm{PaCO}_{2}>45 \mathrm{mmHg}\right)$ & 4,96 & $2,12-11,63$ & 0,001 \\
\hline CVF $<2.840 \mathrm{~mL}$ & 2,84 & $1,45-5,58$ & 0,003 \\
\hline CVF $\leq 90 \%$ teórico & 2,61 & $1,33-5,11$ & 0,005 \\
\hline $\mathrm{VEF}_{1}<800 \mathrm{~mL}$ & 5,32 & $2,30-12,31$ & 0,001 \\
\hline VEF $_{1}<45 \%$ teórico & 2,85 & $1,44-5,64$ & 0,003 \\
\hline $\mathrm{DL}_{\mathrm{co}}<15 \mathrm{~mL} / \mathrm{min} / \mathrm{mmHg}$ & 4,00 & $1,54-4,20$ & 0,005 \\
\hline $\mathrm{DL}_{\mathrm{co}} \leq 50 \%$ teórico & 4,63 & $1,97-10,88$ & 0,001 \\
\hline $\mathrm{DL}_{\mathrm{co}} \mathrm{NA} \leq 4,2 \mathrm{~mL} / \mathrm{min} / \mathrm{mmHg} / \mathrm{L}$ & 5,16 & $1,17-22,80$ & 0,031 \\
\hline $\mathrm{Cl}<2.100 \mathrm{~mL}$ & 3,23 & $1,46-7,16$ & 0,004 \\
\hline $\mathrm{Cl}<80 \%$ teórico & 3,59 & $1,66-7,80$ & 0,002 \\
\hline $\mathrm{CRF}>3.700 \mathrm{~mL}$ & 2,96 & $1,20-7,29$ & 0,018 \\
\hline CRF $>130 \%$ teórico & 2,81 & $1,27-6,25$ & 0,012 \\
\hline $\mathrm{VR}>3.200 \mathrm{~mL}$ & 4,23 & $1,91-9,39$ & 0,001 \\
\hline VR $>160 \%$ teórico & 3,88 & $1,76-8,55$ & 0,001 \\
\hline $\mathrm{Cl} / \mathrm{CPT}<0,33$ & 3,54 & $1,60-7,82$ & 0,002 \\
\hline $\mathrm{CRF} / \mathrm{CPT}>0,62$ & 3,62 & $1,53-8,60$ & 0,004 \\
\hline $\mathrm{VR} / \mathrm{CPT}>0,55$ & 4,92 & $2,20-11,02$ & 0,001 \\
\hline Distancia recorrida en $6 \mathrm{~min}<470 \mathrm{~m}$ & 7,71 & $3,35-17,76$ & 0,001 \\
\hline Distancia recorrida en $6 \min <75 \%$ teórico & 4,75 & $2,33-9,65$ & 0,001 \\
\hline Área de enfisema > 18\% (-960 UH) & 2,55 & $1,06-6,15$ & 0,038 \\
\hline Área de sección del músculo del muslo $<52 \mathrm{~cm}^{2}$ & 2,68 & $1,11-6,48$ & 0,028 \\
\hline
\end{tabular}

Nota: IC95\%: Intervalo de confianza del 95\%; EPOC: Enfermedad pulmonar obstructiva crónica; CVF: Capacidad vital forza$\mathrm{da} ; \mathrm{VEF}_{1}$ : Volumen espiratorio forzado del 1 er segundo; $\mathrm{DL}_{\mathrm{co}}$ : Capacidad de difusión de monóxido de carbono; $V A$ : Volumen alveolar; CPT: Capacidad pulmonar total; Cl: Capacidad inspiratoria; CRF: Capacidad residual funcional; VR: Volumen residual. 
0,71), hiperinsuflación pulmonar (CI/CPT, CRF/ CPT y VR/CPT; AUC: 0,69 a 0,71), $\mathrm{DL}_{\mathrm{CO}}$ (AUC: $0,67)$ y la distancia recorrida en seis minutos (AUC: 0,75) (Tabla 5). El índice pronóstico BODE permitió identificar a los pacientes con mayor riesgo de mortalidad en el seguimiento a cinco años (AUC: 0,68; IC95\% 0,62-0,75; p < 0,001). En la Figura 1 se representa la sobrevida actuarial a cinco años según categorías GOLD de $\mathrm{VEF}_{1}$ (AUC: 0,63; IC95\% 0,56-0,70; p: 0,007); los pacientes con EPOC muy severa $\left(\mathrm{VEF}_{1}<30 \%\right)$ tuvieron menor sobrevida (50\% de mortalidad a cinco años).

Tabla 4. Variables clínicas y funcionales asociadas al riesgo de muerte en el seguimiento a cinco años de una cohorte de pacientes con enfermedad pulmonar obstructiva crónica. Análisis multivariado

\begin{tabular}{|lccccc|}
\hline Factor pronóstico & Coeficiente & Error estándar & Odds ratio & IC 95\% & p \\
\hline Disnea $(\mathrm{mMRC})$ & 0,6184 & 0,1818 & 1,86 & $1,30-2,65$ & 0,001 \\
$\mathrm{PaO}_{2}(\mathrm{mmHg})$ & $-0,0632$ & 0,0315 & 0,94 & $0,88-0,99$ & 0,045 \\
\hline Distancia recorrida en seis minutos $(\mathrm{m})$ & $-0,0072$ & 0,0029 & 0,99 & $0,98-0,99$ & 0,014 \\
\hline
\end{tabular}

Tabla 5. Área bajo la curva receptor operador (AUC) de las variables clínicas y funcionales asociadas al riesgo de muerte en el seguimiento a cinco años de una cohorte de pacientes con enfermedad pulmonar obstructiva crónica

\begin{tabular}{|c|c|c|c|}
\hline Factor pronóstico & AUC & IC $95 \%$ & $\mathbf{p}$ \\
\hline Edad (años) & 0,62 & $0,55-0,69$ & 0,008 \\
\hline Disnea (mMRC) & 0,67 & $0,60-0,74$ & 0,001 \\
\hline $\mathrm{PaO}_{2}(\mathrm{mmHg})$ & 0,71 & $0,64-0,78$ & 0,001 \\
\hline $\mathrm{PaCO}_{2}(\mathrm{mmHg})$ & 0,62 & $0,54-0,69$ & 0,036 \\
\hline CVF $(m L)$ & 0,65 & $0,58-0,71$ & 0,002 \\
\hline CVF (\% teórico) & 0,65 & $0,58-0,72$ & 0,002 \\
\hline $\operatorname{VEF}_{1}(\mathrm{~mL})$ & 0,65 & $0,57-0,71$ & 0,004 \\
\hline $\mathrm{VEF}_{1}$ (\% teórico) & 0,63 & $0,56-0,70$ & 0,012 \\
\hline $\mathrm{DL}_{\mathrm{CO}}(\mathrm{mL} / \mathrm{min} / \mathrm{mmHg})$ & 0,67 & $0,60-0,75$ & 0,002 \\
\hline $\mathrm{DL}_{\mathrm{co}}$ (\% teórico) & 0,69 & $0,61-0,76$ & 0,002 \\
\hline $\mathrm{DL}_{\mathrm{co}} \mathrm{NA}(\mathrm{mL} / \mathrm{min} / \mathrm{mmHg} / \mathrm{L})$ & 0,63 & $0,55-0,71$ & 0,017 \\
\hline $\mathrm{Cl}(\mathrm{mL})$ & 0,66 & $0,58-0,73$ & 0,002 \\
\hline Cl (\% teórico) & 0,65 & $0,57-0,72$ & 0,013 \\
\hline $\mathrm{CRF}(\mathrm{mL})$ & 0,63 & $0,55-0,71$ & 0,014 \\
\hline CRF (\% teórico) & 0,63 & $0,55-0,70$ & 0,027 \\
\hline $\mathrm{VR}(\mathrm{mL})$ & 0,67 & $0,59-0,74$ & 0,003 \\
\hline VR (\% teórico) & 0,62 & $0,54-0,69$ & 0,044 \\
\hline $\mathrm{Cl} / \mathrm{CPT}$ & 0,69 & $0,62-0,76$ & 0,001 \\
\hline CRF/CPT & 0,69 & $0,61-0,76$ & 0,001 \\
\hline VR/CPT & 0,71 & $0,63-0,78$ & 0,001 \\
\hline Distancia recorrida en 6 min (m) & 0,75 & $0,68-0,81$ & 0,001 \\
\hline Distancia recorrida en 6 min (\% teórico) & 0,71 & $0,64-0,77$ & 0,001 \\
\hline Área de enfisema (\% -960 UH) & 0,61 & $0,52-0,69$ & 0,095 \\
\hline Área de sección del músculo del muslo $\left(\mathrm{cm}^{2}\right)$ & 0,64 & $0,55-0,72$ & 0,038 \\
\hline
\end{tabular}

Nota: AUC: Área bajo la curva receptor operador; IC95\%: Intervalo de confianza del 95\%; CVF: Capacidad vital forzada; VEF; Volumen espiratorio forzado del $1^{\text {er }}$ segundo; $\mathrm{DL}_{\mathrm{CO}}$ : Capacidad de difusión de monóxido de carbono; VA: Volumen alveolar; CPT: Capacidad pulmonar total; Cl: Capacidad inspiratoria; CRF: Capacidad residual funcional; VR: Volumen residual. 


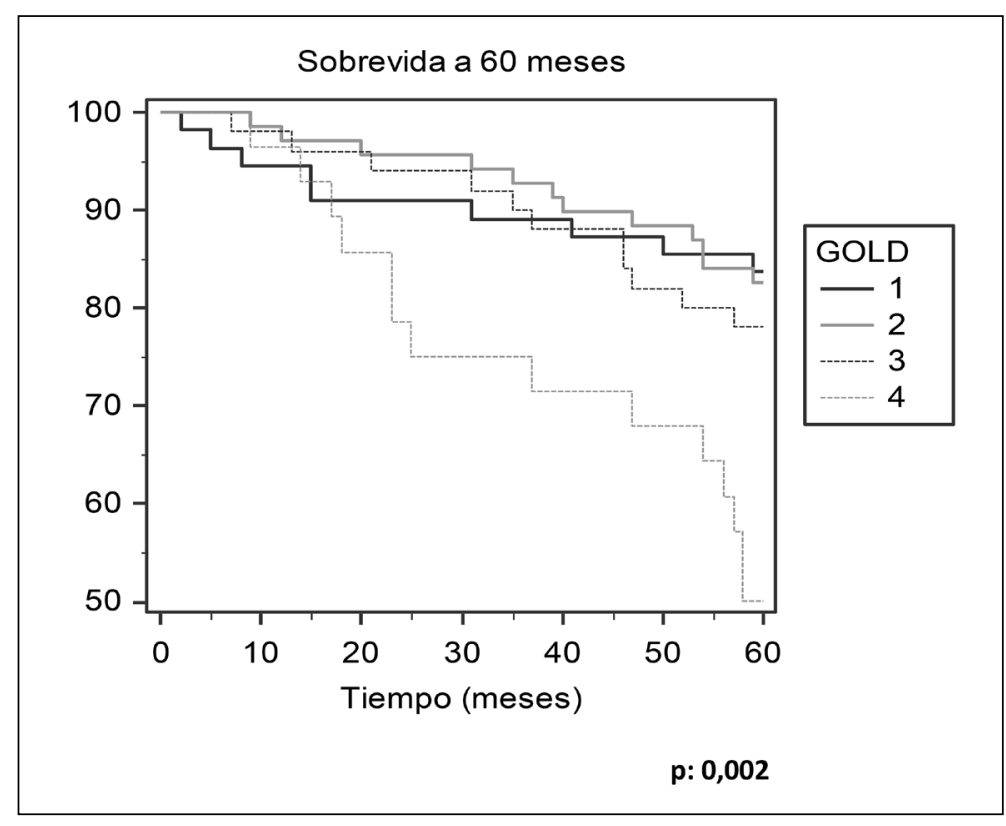

Figura 1. Curvas de sobrevida de Kaplan Meier de pacientes portadores de enfermedad pulmonar obstructiva crónica según categorías GOLD.

\section{Discusión}

Los principales hallazgos de este estudio son: 1) Las variables clínicas asociadas al riesgo de muerte en el seguimiento a largo plazo en pacientes con EPOC son la edad, sexo masculino, magnitud de la disnea, historia de insuficiencia respiratoria crónica o uso de oxígeno domiciliario y antecedente de exacerbaciones graves en los últimos doce meses; 2) Las variables funcionales asociadas al riesgo de muerte en pacientes con EPOC son la magnitud del enfisema pulmonar, reducción de la $\mathrm{DL}_{\mathrm{CO}}$, hiperinsuflación pulmonar, reducción del área de sección transversal del músculo del muslo y la distancia recorrida en seis minutos; 3) El índice multidimensional BODE permite predecir el riesgo de muerte a largo plazo en pacientes con EPOC con bastante precisión.

En nuestra cohorte de seguimiento de pacientes con EPOC hemos observado que el riesgo de muerte a largo plazo (60 meses) estuvo asociado a la edad, sexo masculino, magnitud de la disnea, historia de exacerbaciones graves, insuficiencia respiratoria crónica parcial o global, uso de oxígeno domiciliario, capacidad de ejercicio e índices de hiperinsuflación pulmonar. Los principales predictores de mortalidad en el seguimiento a largo plazo fueron la edad avanzada ( $\geq 65$ años), magnitud de la disnea $(m M R C \geq 2)$, insuficiencia respiratoria crónica $\left(\mathrm{PaO}_{2}<60 \mathrm{mmHg}\right.$ respirando aire ambiente) y reducción de la distancia recorrida en seis minutos; variables clínicas y funcionales que se pueden medir en los servicios de atención primaria y que pueden ser modificadas con el tratamiento. Los fármacos broncodilatadores y la rehabilitación respiratoria alivian la disnea, disminuyen el riesgo de exacerbaciones y aumentan la capacidad de realizar ejercicio ${ }^{31,32}$; mientras que la oxigenoterapia domiciliaria corrige la hipoxemia crónica y mejora la sobrevida de los pacientes con EPOC severa e insuficiencia respiratoria crónica $^{33}$. La evaluación clínica y funcional sistemática en los pacientes con EPOC permite identificar a los grupos de mayor riesgo de morbilidad y mortalidad, en quienes el uso racional de las medidas preventivas y terapéuticas permitiría aliviar la disnea, aumentar la capacidad física y reducir el riesgo de exacerbaciones, mejorando la calidad de vida y sobrevida ${ }^{1}$.

Además de la obstrucción del flujo aéreo expresada mediante la medición de $\mathrm{VEF}_{1}$, se han identificado otros factores predictores del riesgo de morbilidad y mortalidad en pacientes con EPOC, incluyendo la edad ${ }^{34}$, el consumo de tabaco ${ }^{35,36}$, el grado de disnea ${ }^{37}$, la capacidad de ejercicio ${ }^{38}$, el estado nutritivo (índice de masa corporal) ${ }^{39}$, el 
riesgo de exacerbaciones ${ }^{40}$, la comorbilidad cardiovascular ${ }^{41}$ y la calidad de vida ${ }^{42}$. La combinación de varios de estos factores pronósticos en un índice multidimensional incorpora la visión holística actual sobre la EPOC y, en última instancia, puede proporcionar a los médicos una poderosa herramienta para evaluar y controlar la gravedad de la enfermedad para guiar la toma de decisiones y mejorar el resultado del paciente ${ }^{43,44}$. Además de monitorear y guiar la toma de decisiones en el manejo de los pacientes ${ }^{1}$, un índice de pronóstico también podría emplearse para predecir el uso de recursos sanitarios, identificar y enfocarse en grupos particulares de alto riesgo dentro de la población de pacientes con EPOC o para la estratificación de riesgo en los ensayos clínicos.

Se han diseñado varios índices pronósticos que examinan el riesgo de muerte a mediano y largo plazo en pacientes con EPOC (Tabla 6) ${ }^{28,34,45-50}$. Similar a lo descrito en nuestro estudio, la edad ${ }^{34,46,50}$, sexo masculino ${ }^{46}$, magnitud de la disnea ${ }^{28,34,45,47,48,50}$, riesgo de exacerbaciones ${ }^{45-47,50}$, grado de obstrucción bronquial ${ }^{28,34,45-50}$, insuficiencia respiratoria crónica $^{47}$ y limitación de la actividad física ${ }^{28,45,47-49}$ están incorporados en varios índices predictores del riesgo de muerte de los pacientes con EPOC en el seguimiento a mediano y largo plazo. La evaluación de la magnitud de la disnea mediante la escala mMRC, el riesgo de exacerbaciones en los últimos doce meses, las comorbilidades y el grado de obstrucción bronquial objetivado mediante el $\mathrm{VEF}_{1}$ en los servicios de atención primaria, permitiría identificar a los grupos de riesgo, racionalizar las medidas terapéuticas y preventivas dirigidas a esta población, optimizando el uso de los recursos sanitarios ${ }^{1}$.

Las principales limitaciones de nuestro estudio fueron: a) estudio realizado en un servicio de salud docente-asistencial, lo cual limita la generalización de los resultados; b) El diseño del estudio no permitió medir la calidad de vida y biomarcadores relacionados con la sobrevida en pacientes con EPOC $^{46,48,49}$.

En nuestro estudio, similar a lo descrito en los principales modelos predictivos ${ }^{28,34,45-50}$, los principales factores de riesgo de muerte a largo plazo identificados en pacientes con enfermedad pulmonar obstructiva crónica fueron la edad avanzada, magnitud de la disnea, riesgo de exacerbaciones, grado de obstrucción bronquial objetivado mediante la espirometría o medición de volúmenes pulmonares, la presencia de insuficiencia respiratoria crónica o uso de oxígeno domiciliario, la magnitud del enfisema pulmonar objetivado mediante imágenes o reducción de la $\mathrm{DL}_{\mathrm{CO}}$ y la limitación de la actividad física objetiva-

Tabla 6. Índices predictores de mortalidad en el mediano y largo plazo en pacientes con enfermedad pulmonar obstructiva crónica ${ }^{28,34,45-50}$

\begin{tabular}{|c|c|c|c|c|c|c|c|c|c|c|}
\hline $\begin{array}{l}\text { Índice } \\
\text { pronóstico }\end{array}$ & Edad & Sexo & Disnea & IMC & $\begin{array}{l}\text { Exacer- } \\
\text { bación }\end{array}$ & $\begin{array}{l}\text { Comorbillidad } \\
\text { cardiovascular }\end{array}$ & VEF $_{1}$ & $\begin{array}{l}\mathrm{PaO}_{2}^{*} \\
\mathrm{IL}-6^{* *} \\
\mathrm{VI}^{* * *}\end{array}$ & $\begin{array}{l}\text { Ejercicio* } \\
\text { Actividad* } \\
\text { Fuerza*** }\end{array}$ & $\begin{array}{l}\text { Calidad } \\
\text { de vida }\end{array}$ \\
\hline $\mathrm{ADO}^{34}$ & $x$ & & $x$ & & & & $x$ & & & \\
\hline $\mathrm{BODE}^{28}$ & & & $x$ & $x$ & & & $x$ & & $X^{*}$ & \\
\hline BODEx $x^{45}$ & & & $x$ & & $x$ & & $x$ & & $X^{*}$ & \\
\hline e-BODE ${ }^{45}$ & & & $x$ & $x$ & $x$ & & $x$ & & $X^{*}$ & \\
\hline $\mathrm{CPI}^{46}$ & $x$ & $x$ & & $x$ & $x$ & $x$ & $x$ & & & $x$ \\
\hline DOREMI BOX ${ }^{47}$ & & & $x$ & $x$ & $x$ & & $x$ & $X^{*}$ & $X^{*}$ & \\
\hline $\mathrm{HADO}^{48}$ & & & $x$ & & & & $x$ & & $X^{* *}$ & $x$ \\
\hline PILE $^{49}$ & & & & & & & $x$ & $X^{* *}$ & $X^{* * *}$ & \\
\hline TARDIS ${ }^{50}$ & $x$ & & $x$ & $x$ & $x$ & & $x$ & $X^{* * *}$ & & \\
\hline
\end{tabular}

Nota: IMC: Índice de masa muscular; Exacerbación: Tasa de exacerbaciones de la EPOC; VEF : Volumen espiratorio forzado del $1^{\text {er }}$ segundo; IL-6: Interleuquina 6 sérica; VI: Vacuna antiinfluenza; Ejercicio: Distancia recorrida en seis minutos; Actividad: Actividad física medida mediante cuestionario; Fuerza: Medición de la fuerza del músculo extensor de la rodilla; Calidad de vida: Medición de la calidad de vida mediante cuestionarios (SGRQ/CRQ y ad hoc). 
da mediante la prueba de caminata de seis minutos o reducción del área de sección transversal de la musculatura del muslo.

\section{Referencias}

1. Global Initiative for Chronic Obstructive Lung Disease (GOLD). Global strategy for the diagnosis, management, and prevention of chronic obstructive pulmonary disease, 2017. http://www.goldcopd.org

2. Lozano R, Naghavi M, Foreman K, Lim S, Shibuya K, Aboyans V, et al. Global and regional mortality from 235 causes of death for 20 age groups in 1990 and 2010: a systematic analysis for the Global Burden of Disease Study 2010. Lancet 2012; 380: 2095-128.

3. Vos T, Flaxman AD, Naghavi M, Lozano R, Michaud C, Ezzati M, et al. Years lived with disability (YLDs) for 1160 sequelae of 289 diseases and injuries 1990-2010: a systematic analysis for the Global Burden of Disease Study 2010. Lancet 2012; 380: 2163-96.

4. Pleis JR, Lucas JW, Ward BW. Summary health statistics for U.S. adults: National Health Interview Survey, 2008. Vital Health Stat 2009; 10: 1-157.

5. Menezes AM, Pérez-Padilla R, Jardim JR, Muiño A, Lopez MV, Valdivia G, et al; PLATINO Team. Chronic obstructive pulmonary disease in five Latin American cities (the PLATINO study): a prevalence study. Lancet 2005; 366: 1875-81.

6. Zhou X, Li Q, Zhou X. Exacerbation of Chronic Obstructive Pulmonary Disease. Cell Biochem Biophys 2015; 73: 349-55.

7. Anthonisen NR, Wright EC, Hodgkin JE, IPPB Trial Group. Prognosis in chronic obstructive pulmonary disease. Am Rev Respir Dis 1986; 133: 14-20.

8. Mannino DM, Reichert MM, Davis KJ. Lung function decline and outcomes in an adult population. Am J Respir Crit Care Med 2006; 173: 985-90.

9. Hoogendoorn M, Feenstra TL, Hoogenveen RT, Al M, Mölken MR. Association between lung function and exacerbation frequency in patients with COPD. Int J Chron Obstruct Pulmon Dis 2010; 5: 435-44.

10. Tashkin DP, Celli B, Senn S, Burkhart D, Kesten S, Menjoge S, et al. A 4-year trial of tiotropium in chronic obstructive pulmonary disease. N Engl J Med 2008; 359 : 1543-54.

11. Yang IA, Clarke MS, Sim EH, Fong KM. Inhaled corticosteroids for stable chronic obstructive pulmonary disease. Cochrane Database Syst Rev 2012; 7: CD002991.

12. Calverley PM, Anderson JA, Celli B, Ferguson GT, Jenkins C, Jones PW, et al. Salmeterol and fluticasone propionate and survival in chronic obstructive pulmonary disease. N Engl J Med 2007; 356: 775-89.

13. van den Bemt L, Schermer TR. Multicomponent staging indices for COPD in daily patient care: what's the yield? Int J Clin Pract 2010; 64: 1475-9.

14. Mannino DM, Buist AS. Global burden of COPD: risk factors, prevalence, and future trends. Lancet 2007; 370: 765-73.

15. Schunemann H. From BODE to ADO to outcomes in multimorbid COPD patients. Lancet 2009; 374: 667 8.

16. Han MK, Agusti A, Calverley PM, Celli BR, Criner G, Curtis JL, et al. Chronic obstructive pulmonary disease phenotypes: the future of COPD. Am J Respir Crit Care Med 2010;182:598-604.

17. Berry CE, Wise RA. Mortality in COPD: causes, risk factors, and prevention. COPD 2010; 7: 375-82.

18. Mahler DA, Wells CK. Evaluation of clinical methods for rating dyspnea. Chest 1988; 93: 580-6.

19. Burge S, Wedzicha JA. COPD exacerbations: definitions and classifications. Eur Respir J 2003; 41 (Suppl): 46S-53S.

20. Miller MR, Hankinson J, Brusasco V, Burgos F, Casaburi $\mathrm{R}$, Coates A, et al. Standardisation of spirometry. Eur Respir J 2005; 26: 319-38.

21. Hankinson JL, Odencrantz JR, Fedan KB. Spirometric reference values from a sample of the general U.S. population. Am J Respir Crit Care Med 1999; 159: 179-87.

22. Wanger J, Clausen JL, Coates A, Pedersen OF, Brusasco $\mathrm{V}$, Burgos F, et al. Standardisation of the measurement of lung volumes. Eur Respir J 2005; 26: 511-22.

23. MacIntyre N, Crapo RO, Viegi G, Johnson DC, van der Grinten CP, Brusasco V, et al. Standardisation of the single-breath determination of carbon monoxide uptake in the lung. Eur Respir J 2005; 26: 720-35.

24. Cotes JE, Chinn DJ, Quanjer PH, Roca J, Yernault JC. Standardization of the measurement of transfer factor (diffusing capacity). Report Working Party Standardization of Lung Function Tests, European Community for Steel and Coal. Official Statement of the European Respiratory Society. Eur Respir J 1993; 16 (Suppl): 41 52.

25. American Thoracic Society Statement. Guidelines for the six-minute walk test. Am J Respir Crit Care Med 2002; 166: 111-7.

26. Troosters T, Gosselink R, Decramer M. Six minute walking distance in healthy elderly subjects. Eur Respir J 1999; 14: 270-4.

27. Borg GA. Psychophysical basis of perceived exertion. Med Sci Sports Exerc 1982; 14: 377-81.

28. Celli BR, Cote CG, Marín JM, Casanova C, Montes de 
Oca M, Méndez RA, et al. The body-mass index, airflow obstruction, dyspnea and exercise capacity index in chronic obstructive pulmonary disease. N Engl J Med 2004; 350: 1005-12.

29. Díaz AA, Morales A, Díaz JC, Ramos C, Klaassen J, Saldias F, et al. CT and physiologic determinants of dyspnea and exercise capacity during the six-minute walk test in mild COPD. Respir Med 2013; 107: 570-9.

30. Bernard S, LeBlanc P, Whittom F, Carrier G, Jobin J, Belleau R, et al. Peripheral muscle weakness in patients with chronic obstructive pulmonary disease. Am J Respir Crit Care Med 1998; 158: 629-34.

31. Singh D. New combination bronchodilators for chronic obstructive pulmonary disease: current evidence and future perspectives. Br J Clin Pharmacol 2015; 79: 695708.

32. Spruit MA, Singh SJ, Garvey C, ZuWallack R, Nici L, Rochester C, et al. An official American Thoracic Society/European Respiratory Society statement: key concepts and advances in pulmonary rehabilitation. Am J Respir Crit Care Med 2013; 188: e13-64.

33. Barjaktarevic I, Cooper CB. Supplemental oxygen therapy for patients with chronic obstructive pulmonary disease. Semin Respir Crit Care Med 2015; 36: 552-66.

34. Puhan MA, García-Aymerich J, Frey M, ter Riet G, Anto JM, Agusti AG, et al. Expansion of the prognostic assessment of patients with chronic obstructive pulmonary disease: the updated BODE index and the ADO index. Lancet 2009; 374: 704-11.

35. Ezzati M, López AD. Estimates of global mortality attributable to smoking in 2000. Lancet 2003; 362: 847-52.

36. Godtfredsen NS, Lam TH, Hansel TT, Leon ME, Gray $\mathrm{N}$, Dresler C, et al. COPD-related morbidity and mortality after smoking cessation: status of the evidence. Eur Respir J 2008; 32: 844-53.

37. Nishimura K, Izumi T, Tsukino M, Oga T. Dyspnea is a better predictor of 5-year survival than airway obstruction in patients with COPD. Chest 2002; 121: 1434-40.

38. Oga T, Nishimura K, Tsukino M, Sato S, Hajiro T. Analysis of the factors related to mortality in chronic obstructive pulmonary disease: role of exercise capacity and health status. Am J Respir Crit Care Med 2003; 167: 544-9.
39. Landbo C, Prescott E, Lange P, Vestbo J, Almdal TP. Prognostic value of nutritional status in chronic obstructive pulmonary disease. Am J Respir Crit Care Med 1999; 160: 1856-61.

40. Donaldson GC, Wedzicha JA. COPD exacerbations 1: Epidemiology. Thorax 2006; 61: 164-8.

41. Mannino DM, Thorn D, Swensen A, Holguin F. Prevalence and outcomes of diabetes, hypertension and cardiovascular disease in COPD. Eur Respir J 2008; 32 : 962-9.

42. Fan VS, Curtis JR, Tu SP, McDonell MB, Fihn SD; Ambulatory Care Quality Improvement Project Investigators. Using quality of life to predict hospitalization and mortality in patients with obstructive lung diseases. Chest 2002; 122: 429-36.

43. Schunemann H. From BODE to ADO to outcomes in multimorbid COPD patients. Lancet 2009;374:667-8.

44. Moons KG, Royston P, Vergouwe Y, Grobbee DE, Altman DG. Prognosis and prognostic research: what, why, and how? BMJ 2009; 338: b375.

45. Soler-Cataluña JJ, Martínez-García MA, Sánchez LS, Tordera MP, Sánchez PR. Severe exacerbations and BODE index: two independent risk factors for death in male COPD patients. Respir Med 2009; 103: 692-9.

46. Briggs A, Spencer M, Wang H, Mannino D, Sin DD. Development and validation of a prognostic index for health outcomes in chronic obstructive pulmonary disease. Arch Intern Med 2008; 168: 71-9.

47. Kostianev SS, Hodgev VA, Iluchev DH. Multidimensional system for assessment of COPD patients. Comparison with BODE index. Folia Med (Plovdiv) 2008; 50: 29-38.

48. Esteban C, Quintana JM, Aburto M, Moraza J, Capelastegui A. A simple score for assessing stable chronic obstructive pulmonary disease. QJM 2006; 99: 751-9.

49. Mehrotra N, Freire AX, Bauer DC, Harris TB, Newman $A B$, Kritchevsky SB, et al. Predictors of mortality in elderly subjects with obstructive airway disease: the PILE score. Ann Epidemiol 2010; 20: 223-32.

50. Schembri S, Anderson W, Morant S, Winter J, Thompson P, Pettitt D, et al. A predictive model of hospitalisation and death from chronic obstructive pulmonary disease. Respir Med 2009; 103: 1461-7. 